\title{
Investigating stable chest pain of suspected cardiac origin
}

The authors of this Practice article (BMJ 2013;347:f3940, doi:10. 1136/bmj.f3940), Declan O'Regan and colleagues, have informed us that the definition of "High risk" in the table was incorrect and should have read: "High risk=diabetes, smoking, and [not "or" as published] hyperlipidaemia." That is, all three factors, not just one, need to be present to be considered high risk.

Cite this as: BMJ 2013;346:f7573

๑ BMJ Publishing Group Ltd 2013 